\title{
Variación ambiental y patrones dietarios del erizo negro Tetrapygus niger en costas intermareales rocosas del norte de Chile
}

\author{
Environmental variation and dietary patterns of the black urchin Tetrapygus niger in rocky \\ intertidal shores of northern Chile
}

\author{
ARTURO H. NAVARRETE ${ }^{1}$, PATRICIO A. CAMUS ${ }^{1,2, *}$ \& L. FELIPE OPAZO ${ }^{1}$ \\ ${ }^{1}$ Departamento de Ecología Costera, Facultad de Ciencias, Universidad Católica de la Santísima Concepción, \\ Casilla 297, Concepción, Chile \\ ${ }^{2}$ Center for Advanced Studies in Ecology and Biodiversity \\ *e-mail para correspondencia: pcamus@ucsc.cl
}

\begin{abstract}
RESUMEN
El rol e impacto de los herbívoros en la trama trófica de comunidades intermareales rocosas podría ser más diverso y complejo de lo considerado hasta ahora, particularmente en el caso de consumidores de mayor tamaño y abundancia como el erizo negro Tetrapygus niger (Molina) en la costa de Chile. En este contexto, analizamos la variación en riqueza y composición de la dieta de $T$. niger en cuatro comunidades intermareales rocosas del norte de Chile, distribuidas en $1.000 \mathrm{~km}$ de costa, y muestreadas estacionalmente entre invierno 2004 y primavera 2005. Durante el estudio ocurrió el evento El Niño 2004-2005 cuyo efecto potencial fue incorporado en esta evaluación. En cada comunidad y estación se evaluó la abundancia de las especies presentes en 45 cuadrantes $\left(0,25 \mathrm{~m}^{2}\right)$ distribuidos en cinco bloques permanentes, recolectando cerca de 10 individuos de $T$. niger a los que se midió su biomasa y longitud, determinando el número, identidad y frecuencia de ocurrencia de ítemes en su contenido gástrico. Colectamos un total de 251 individuos de $T$. niger, que en conjunto consumieron 89 ítemes de algas (64\%) e invertebrados (36\%; incluyendo otros herbívoros). Una relación inversa significativa entre el tamaño corporal y el número de ítemes animales ingeridos sugiere que $T$. niger varía su dieta durante la ontogenia. La amplitud de nicho dietario fue alta y tendió a disminuir hacia latitudes menores. Globalmente, el espectro y frecuencia de ocurrencia de ítemes dietarios no fueron significativamente distintos entre comunidades, pese a marcadas diferencias en intensidad de surgencia entre ellas. Similarmente, no hubo relación clara entre la oferta ambiental de recursos y su frecuencia en la dieta de T. niger, destacando la clorófita Ulva rigida que fue el ítem consumido con más frecuencia en cada comunidad pero no el más abundante en terreno. Sin embargo, la riqueza dietaria de $T$. niger se relacionó negativa y significativamente con la temperatura superficial del mar, y fue significativamente menor en todo el período de El Niño 2004-2005. Nuestros datos sugieren que T. niger es un generalista polífago cuyos patrones de consumo son fuertemente modulados por la fluctuación térmica ambiental. Además, hipotetizamos que T. niger puede usar estrategias oportunistas y selectivas sobre distintos recursos, y que su conectividad y posición trófica serían más complejas que las de un simple consumidor primario.
\end{abstract}

Palabras clave: herbivoría, amplitud de nicho, omnivoría, surgencia, El Niño.

\begin{abstract}
The role and impact of herbivores on rocky intertidal food webs could be more complex and diverse than previously considered, particularly in the case of larger and more abundant consumers such as the black urchin Tetrapygus niger (Molina) in the coast of Chile. In this context, we analyzed the variation in diet richness and composition of the black urchin in four rocky intertidal communities over a $1,000 \mathrm{~km}$ stretch of coast in northern Chile, sampled seasonally between the austral winter of 2004 and spring of 2005. The 2004-2005 El Niño event took place during the study, and we incorporated its potential effects to this evaluation. For each community and season, we measured the abundance of species present in 45 quadrants $\left(0.25 \mathrm{~m}^{2}\right)$ distributed between five permanent blocks, collecting about 10 individuals of $T$. niger to measure their body size (dry biomass and maximum diameter) and to determine the number, identity and occurrence frequency of dietary items in their gastric tract. We collected a total of 251 urchin individuals, which consumed 89 items of algae (64\%) and invertebrates (36\%; including other herbivores). A significant inverse relationship between the number of ingested animal items and body size suggests that $T$. niger
\end{abstract}


changes its diet throughout the ontogeny. Dietary niche breadth was high but tended to decrease toward lower latitudes. The spectrum and occurrence frequency of dietary items were not significantly different among communities, despite their marked differences in upwelling intensity. Similarly, we did not find clear relationships between the natural abundance of resources and their frequency in the diet of $T$. niger, highlighting the green alga Ulva rigida which was the most frequently consumed item in all communities although not the most abundant in the field. However, the dietary richness of $T$. niger showed a significant negative relation with sea surface temperature, and was significantly lower throughout the El Niño period. Our data suggest that $T$. niger is a polyphagous generalist whose consumption patterns are strongly modulated by environmental thermal fluctuations. We also hypothesize that T. niger could use both opportunistic and selective strategies on different resources, and its trophic position and connectedness would be more complex than those of a simple primary consumer.

Key words: herbivory, niche breadth, omnivory, upwelling, El Niño.

\section{INTRODUCCIÓN}

Los equinoídeos son herbívoros comunes, eficientes y ecológicamente importantes en todos los sistemas marinos litorales del mundo, ejerciendo efectos significativos sobre los ensambles de algas en ambientes intermareales y submareales (Lawrence 1975, Castilla \& Moreno 1982, Hawkins \& Hartnoll 1983, Santelices \& Ojeda 1984, Harold \& Pearse 1987, Vásquez \& Buschmann 1997). En Chile, sus dos representantes más conspicuos, ambos encontrados desde Arica hasta el Estrecho de Magallanes, son el erizo negro Tetrapygus niger (Molina, 1782), sin valor comercial y con una amplia ocurrencia y abundancia, y el erizo rojo Loxechinus albus (Molina, 1782), intensamente explotado en toda la costa por lo cual es menos abundante y frecuente (Stotz et al. 1992, Castilla \& Fernández 1998, Arana 2005).

En particular, T. niger es un herbívoro de amplio espectro dietario capaz de consumir algas tanto fijas en el substrato como a la deriva (Contreras \& Castilla 1987, Rodríguez 2003), y su alta densidad e intenso pastoreo pueden generar y mantener amplias zonas desprovistas de vegetación ("barren grounds", Vásquez 2000). Sin embargo, pese a la gran importancia ecológica de T. niger, aún no se cuenta con un registro detallado de la riqueza y composición de su dieta, especialmente en la zona intermareal rocosa. Más aún, aunque la mayoría de los erizos son funcionalmente herbívoros, muchas especies consumen regularmente presas animales y la evidencia indica que pueden ser omnívoras de forma permanente o facultativa (e.g., Lawrence \& Sammarco 1982, Briscoe \& Sebens 1988, Hughes et al. 2005, Knip \& Scheibling 2007).
La potencial omnivoría de $T$. niger es un aspecto poco conocido, que hasta ahora solo ha sido inferido o documentado de forma indirecta (e.g., Vásquez et al. 2006, Fariña et al. en prensa). Si bien no existen evaluaciones de la capacidad de $T$. niger para digerir y asimilar alimento animal, antecedentes recientes muestran que esta especie ingiere ítemes animales en forma frecuente y consistente, incluyendo a otros herbívoros, lo cual abre numerosas perspectivas sobre su rol potencial como omnívoro y en procesos de depredación intragremios (Camus et al. 2008). En este contexto, un conocimiento más preciso de la dieta de especies como el erizo negro resulta esencial para clarificar su grado de conectividad y posición trófica en los flujos de energía de la trama intermareal.

Por otra parte, la dieta de un herbívoro es susceptible a las fluctuaciones físicas y biológicas que afectan la calidad o disponibilidad de sus recursos, en general asociadas a la variación en productividad primaria (e.g., Bustamante et al. 1995), o entre otros aspectos a interacciones entre consumidores (e.g., Contreras \& Castilla 1987). En Chile estos procesos se vinculan especialmente a variaciones de mesoescala en la intensidad de surgencia y suministro de nutrientes, que inciden diferencialmente en el crecimiento algal y pueden determinar el impacto de un herbívoro en la comunidad (Nielsen \& Navarrete 2004), o a la variación interanual ligada a los eventos El Niño y La Niña, capaz a su vez de modificar la intensidad y efectos de la surgencia (véase revisión de Thiel et al. 2007). Estos factores son de particular relevancia en la costa norte de Chile, donde sus efectos interactúan de forma compleja afectando a las poblaciones de 
herbívoros y sus patrones de consumo de recursos (e.g., Vega et al. 2005, Thiel et al. 2007, Camus 2008).

En este contexto, presentamos una evaluación de la riqueza y composición dietaria de Tetrapygus niger en la zona intermareal rocosa de cuatro localidades del norte de Chile muestreadas estacionalmente entre invierno 2004 y primavera 2005, analizando la relación de la dieta con factores biológicos como el tamaño corporal y la oferta ambiental de recursos, y factores físicos como la temperatura superficial del mar. Además discutimos los posibles efectos del evento El Niño 2004-2005 ocurrido durante el período abarcado por este trabajo (CPC 2007), sobre la variación espaciotemporal de la dieta de T. niger.

\section{MATERIALES Y MÉTODOS}

\section{Obtención de datos}

El presente trabajo forma parte de un estudio más amplio de la diversidad y estructura de comunidades en costas rocosas del norte de Chile, que incluye un análisis trófico detallado de varias especies de consumidores (e.g., Camus et al. 2008). Para ello seleccionamos cuatro comunidades intermareales rocosas (Fig. 1), abarcando ca. $10^{\circ}$ de latitud: Río Seco ( $21^{\circ} 00^{\prime} 05^{\prime}$ ' S, 7009' $54^{\prime}$ ' O, al sur de Iquique),

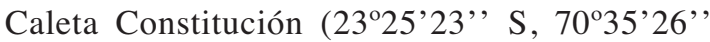
O, costa oeste de la Península de Mejillones), Caleta Angosta $\left(28^{\circ} 15^{\prime} 38^{\prime \prime} \mathrm{S}, 71^{\circ} 10^{\prime} 23^{\prime}\right.$ ' O, al norte de Huasco), y Lagunillas (30 $06^{\prime} 14^{\prime}$ ' S, 71'22'57' O, al sur de Coquimbo). Los datos informados en este trabajo provienen de seis muestreos estacionales realizados en los mismos períodos en las cuatro comunidades: invierno 2004 (15-30 agosto), primavera 2004 (8-15 noviembre), verano 2005 (20-26 enero), otoño 2005 (22-28 mayo), invierno 2005 (18-24 agosto), primavera 2005 (8-15 noviembre). En cada comunidad y estación, durante los períodos de marea baja se evaluó la abundancia de especies en 45 cuadrantes de $0,25 \mathrm{~m}^{2}(50 \mathrm{x}$ $50 \mathrm{~cm}$ ), registrando en cada cuadrante la densidad de organismos móviles por conteo directo y el porcentaje de cobertura de organismos sésiles por su ubicación en 100 puntos de intersección. Los cuadrantes de muestreo se distribuyeron en cinco bloques permanentes (nueve por bloque) espaciados cada $15 \mathrm{~m}$ y con su ubicación georreferenciada. Cada bloque incluyó tres series de cuadrantes dispuestos en las zonas intermareales alta, media y baja (tres por zona), espaciados entre sí cada 1,5 y $5 \mathrm{~m}$ en sentido vertical y horizontal respectivamente. En cada muestreo se registró la temperatura superficial del mar (en adelante TSM) con data loggers (Onset Stowaway) instalados en forma permanente en cada localidad (sumergidos $1 \mathrm{~m}$ bajo MLLW frente al sector central de la franja de costa muestreada). Como precaución adicional se obtuvieron medidas manuales directas en cada día de muestreo mediante un termómetro $( \pm 0,1$ $\left.{ }^{\circ} \mathrm{C}\right)$ tomadas en la misma zona del data logger. Se informa el promedio de los valores de TSM de las fechas de muestreo en cada localidad.

Para análisis de contenidos gástricos, en base a estimaciones preliminares se planeó colectar al azar 10 individuos de $T$. niger por estación y localidad, número que permitía representar entre 85 y $100 \%$ de los ítemes consumidos localmente en todas las estaciones (Camus et al. 2008), cercano a lo documentado por otros autores en estudios similares (e.g., Winemiller et al. 2001). En promedio, el número real colectado fue de 10,42 \pm 0,29 ( \pm EE) individuos por localidad y estación (rango: 8-15), los que fueron tomados desde los cinco bloques de muestreo en las zonas mareales media y baja (la especie no se registró en sectores altos) a fin de incorporar la variación espacial de sus recursos tróficos. En terreno cada individuo fue inyectado con formaldehído $10 \%$ para preservar su contenido gástrico, y en laboratorio se estimó su biomasa (peso seco) y longitud (diámetro máximo de testa), previa extracción de su contenido gástrico por disección para ser analizado bajo microscopio y lupa estereoscópica. Los contenidos gástricos fueron identificados al nivel taxonómico más bajo posible y clasificados como ítemes dietarios, cuya presencia fue registrada para cada individuo por separado.

\section{Análisis}

Los análisis descritos a continuación se basan en datos de dieta y de terreno obtenidos por separado, que pueden evaluarse a múltiples escalas y que en algunos casos no tienen una correspondencia uno a uno. En función de las 
variables a evaluar, se han seleccionado combinaciones de datos y niveles de análisis abarcando aspectos regionales y locales para proveer una adecuada caracterización dietaria. En todos los análisis de varianza (ANOVA) se revisó previamente el cumplimiento de homocedasticidad y normalidad mediante distintas pruebas, indicando aquellos casos en que debió efectuarse una transformación correctiva de los datos. Las comunidades de estudio fueron elegidas por sus características contrastantes y para abarcar las zonas representativas del norte de Chile, por lo cual se consideraron un efecto fijo en ANOVA, a diferencia de las estaciones muestreadas que fueron definidas como efecto aleatorio.

Las variaciones en biomasa y longitud entre los individuos de $T$. niger colectados en las cuatro comunidades fueron evaluadas mediante ANOVA de una vía, usando una prueba de Tukey para comparación a posteriori, y adicionalmente se estimó la relación entre biomasa y longitud mediante un ajuste de regresión. La densidad en terreno de $T$. niger fue comparada entre comunidades mediante la prueba de Kruskal-Wallis (KW) debido a la falta de normalidad y homocedasticidad de los datos, aplicando una prueba a posteriori para comparación de medias obtenidas por ranking (Siegel \& Castellan 1988).

De la identificación de contenidos gástricos se obtuvo el número de ítemes dietarios (riqueza dietaria en adelante) consumido por cada individuo analizado, a partir del cual se calculó la frecuencia de ocurrencia de cada ítem en el conjunto de individuos muestreados por comunidad (i.e., número de individuos que consumió un ítem dado respecto al total de individuos muestreados en una comunidad dada, expresado en porcentaje) (véase también Camus et al. 2008). Las variaciones entre comunidades y entre ítemes fueron evaluadas mediante una prueba de Friedman (Fr) de dos vías. En base a valores promedio calculados

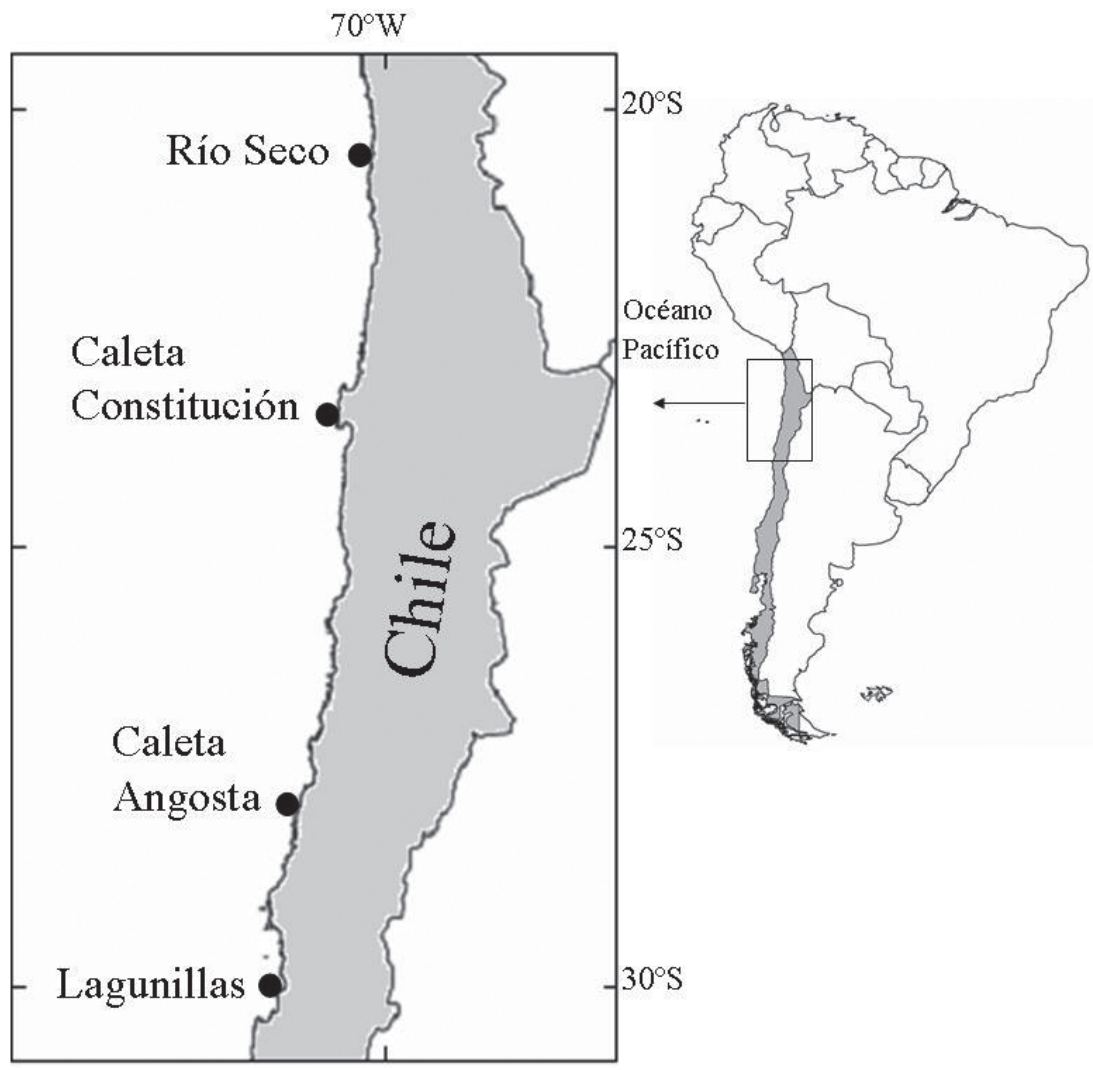

Fig. 1: Localización de las comunidades de estudio en el norte de Chile.

Location of study communities in northern Chile. 
por estación y comunidad, se evaluó la relación de la biomasa y longitud de los individuos de $T$. niger con su riqueza dietaria mediante análisis de correlación de Spearman $\left(\mathrm{r}_{\mathrm{s}}\right)$, considerando tanto el conjunto total de ítemes como los ítemes algales y animales por separado. La variación de la riqueza dietaria por individuo entre comunidades (efecto fijo) y estaciones (efecto aleatorio) se evaluó mediante ANOVA de dos vías, usando la transformación $\sqrt{(\mathrm{x}+1)}$ y aplicando la prueba de Tukey para comparación de medias. La relación global entre la TSM y la riqueza dietaria se evaluó mediante correlación de Spearman usando los valores promedio por estación.

Para cada grupo de individuos de $T$. niger colectados en cada estación y comunidad, se estimó su amplitud de nicho trófico mediante el índice de Levins (recíproco del índice de Simpson, Levins 1968) usando la riqueza y frecuencia de ocurrencia de los ítemes dietarios registrados. La variación en amplitud de nicho entre comunidades se analizó mediante ANOVA de una vía, en base a los valores respectivos de amplitud de nicho por estación.

No fue posible evaluar la correspondencia exacta entre los recursos del ambiente y aquellos consumidos por $T$. niger, dado que estos últimos incluyen especies de pequeño tamaño que no se registran en los muestreos de abundancia in situ, pero no obstante se efectuaron dos aproximaciones. La primera se basó en comparar la frecuencia de ocurrencia en la dieta con la cobertura en terreno para ítemes dietarios particulares de distintos grupos taxonómicos, seleccionados por presentar alta importancia relativa a nivel regional, y por tener disponible su abundancia en terreno en todas las estaciones de muestreo. Estos ítemes fueron Ulva rigida y Enteromorpha sp. entre las clorófitas, Gelidium chilense y coralina incrustante entre las rodófitas, y cirripedios entre los invertebrados. Las feófitas fueron excluidas por su baja representación en el intermareal (e.g., Macrocystis) o en el muestreo. Los datos de terreno para las especies de Enteromorpha y cirripedios fueron agrupados para corresponderse con los ítemes dietarios, y la relación entre importancia en terreno y en la dieta se evaluó mediante correlación de Spearman. La segunda aproximación evaluó la relación entre el porcentaje promedio de cobertura en el ambiente y la frecuencia de ocurrencia, ambas a nivel regional, entre todas las especies sésiles registradas tanto en la dieta como en muestreos de terreno. La cobertura de cada especie fue calculada solo desde los cuadrantes en que estuvo presente, excluyendo aquellas especies registradas en solo tres cuadrantes o menos.

Por último, la variación espacial y temporal en la composición de la dieta de $T$. niger se evaluó mediante análisis nMDS seguido de ANOSIM para evaluar diferencias entre comunidades y estaciones, incluyendo un tercer factor para comparar los datos de combinaciones muestrales de comunidad $\mathrm{x}$ estación registrados en presencia versus ausencia del evento El Niño 2004-2005.

\section{RESULTADOS}

\section{Aspectos generales}

Debido a que $T$. niger se ubica preferentemente en niveles mareales bajos, fue registrado en ca. $7 \%$ del total de cuadrantes (71 de 1.080) evaluados en los muestreos comunitarios en el norte de Chile. De ellos, 94,4 \% estaban ubicados en la zona intermareal baja y 5,6 \% en la zona intermareal media. Consecuentemente, la densidad de $T$. niger (Tabla 1) fue evaluada usando solo los cuadrantes de las zonas media y baja, encontrando valores bajos pero significativamente distintos entre comunidades $(\mathrm{KW}=28,72 ; \mathrm{gl}=3 ; \mathrm{n}=712 ; \mathrm{P}<0,0001)$. Sin embargo la comparación pareada a posteriori no detectó diferencias, sugiriendo que la significancia original pudo deberse al efecto del tamaño de muestra sobre la potencia de la prueba, y por tanto que las densidades registradas son más bien similares.

Para el análisis de contenidos gástricos se colectó un total de 251 individuos de $T$. niger en todas las comunidades y estaciones muestreadas. La Tabla 1 muestra las estimaciones del tamaño promedio de los individuos por comunidad, donde se detectaron diferencias significativas tanto en longitud $\left(\mathrm{F}_{3,247}=7,47 ; \mathrm{P}<0,00001\right)$ como en biomasa $\left(\mathrm{F}_{3,247}=10,74 ; \mathrm{P}<0,00001\right)$, en ambos casos con valores significativamente más altos en Caleta Constitución y Caleta Angosta (prueba de Tukey, $\mathrm{P}<0,05$ en todas las comparaciones). Globalmente, la biomasa (B) y 
la longitud (L) estuvieron estrechamente relacionadas por la función potencial $\mathrm{B}=$ $0,0006 \mathrm{~L}^{2,6183}\left(\mathrm{R}^{2}=0,85 ; \mathrm{n}=251 ; \mathrm{P}<0,0001\right)$, indicando que las dos variables son buenas descriptoras del tamaño corporal.

\section{Riqueza y amplitud dietaria}

A nivel regional, la dieta de $T$. niger estuvo compuesta por un total de 89 ítemes (Tabla 2), distribuidos en 32 (36\%) ítemes invertebrados y $57(64 \%)$ ítemes algales, estos últimos distribuidos a su vez en 14,7 \% de clorófitas, $15,7 \%$ de feófitas y $33,7 \%$ de rodófitas. La Tabla 2 presenta los ítemes dietarios registrados y su frecuencia de ocurrencia tanto a nivel regional como en cada comunidad estudiada, incluyendo la cobertura promedio a nivel regional estimada en terreno para 47 especies de algas e invertebrados sésiles que también fueron registradas en la dieta. Entre los taxa algales, algunos de los órdenes y sus miembros más representados fueron: (a) Chlorophyta: Caulerpales (Codium), Cladophorales (Chaetomorpha) y Ulvales (Ulva, Enteromorpha); (b) Phaeophyta: Ectocarpales (Ectocarpus), Laminariales (Lessonia, Macrocystis) y Scytosiphonales (Colpomenia, Halopteris); y (c) Rhodophyta: Ceramiales (Ceramium, Polysiphonia), Cryptonemiales (coralinas incrustantes en general), Gelidiales (Gelidium), Gigartinales (Gastroclonium, Hypnea, Schottera) e
Hildenbrandiales (Hildenbrandia). Entre los taxa animales, los cirripedios fueron claramente mayoritarios (como ítem general y como especies), seguidos por crustáceos (Liopetrolisthes), esponjas (Demospongiae) y mitílidos (Semimytilus). Debe notarse que entre los ítemes de menor importancia estuvieron también los herbívoros Tegula atra, Prisogaster niger, Diloma nigerrima y especies del género Scurria, lo cual puede tener implicancias ecológicas significativas. En general, la importancia de los ítemes principales fue aproximadamente similar a nivel comunitario y regional, y de hecho al analizar el espectro dietario completo no hubo diferencias significativas entre comunidades $(\mathrm{Fr}=5,34 ; \mathrm{gl}=3 ; \mathrm{P}=0,148)$, aunque sí entre ítemes dietarios $(\mathrm{Fr}=219,8 ; \mathrm{gl}=88 ; \mathrm{P}<$ $0,0001)$ debido a su alta diversidad y variabilidad en frecuencia de ocurrencia.

La amplitud del nicho trófico de $T$. niger fue en general muy alta (Tabla 1), con un valor regional (integrando todos los datos obtenidos) de 27,3 no muy distinto a los valores estimados a nivel comunitario (integrando los datos estacionales), los que mostraron una tendencia creciente hacia latitudes más altas, desde 19,51 en Río Seco a 26,95 en Lagunillas. En cada comunidad, la media de los valores de amplitud por estación (Tabla 1) fue menor, evidenciando variaciones temporales importantes, y fluctuó entre 11,75 en Río Seco y 15,94 en Lagunillas con la misma tendencia anterior. Sin embargo,

Atributos básicos (media \pm EE) de Tetrapygus niger en la zona intermareal del norte de Chile, evaluados a nivel comunitario y regional. La amplitud de nicho dietario (índice de Levins) por comunidad se estimó como valor promedio estacional, y como valor global (estaciones agrupadas; entre paréntesis)

Basic attributes (mean $\pm \mathrm{SE}$ ) of Tetrapygus niger in the intertidal zone of northern Chile, evaluated at community and regional levels: number of sampled individuals, density, body size (test diameter and dry biomass), and niche breadth

(Levins' index) per community estimated as mean seasonal value and overall value (pooled seasons in parentheses)

\begin{tabular}{lccccc}
\hline & Río Seco & Caleta Constitución & Caleta Angosta & Lagunillas & Regional \\
\hline Número de individuos & 62 & 65 & 60 & 64 & 251 \\
Densidad (ind m ${ }^{-2}$ ) & $2,19 \pm 0,54$ & $2,3 \pm 0,45$ & $0,04 \pm 0,03$ & $0,79 \pm 0,19$ & $1,33 \pm 0,26$ \\
Diámetro de testa (mm) & $48,6 \pm 1,36$ & $56 \pm 1,32$ & $55,3 \pm 1,81$ & $49,6 \pm 1,5$ & $52,36 \pm 0,77$ \\
Biomasa seca (g) & $16,77 \pm 1,15$ & $26,01 \pm 1,44$ & $26,95 \pm 2,08$ & $18,30 \pm 1,62$ & $21,97 \pm 0,84$ \\
Amplitud de nicho & $11,75 \pm 2,02(19,51)$ & $13,08 \pm 2,02(23,78)$ & $14,80 \pm 2,80(25,06)$ & $15,94 \pm 2,29(26,95)$ & 27,3 \\
\end{tabular}




\section{TABLA 2}

Ítemes dietarios de Tetrapygus niger en el norte de Chile, separados por grupo taxonómico y listados en orden decreciente según su frecuencia de ocurrencia regional. Columna dos: abundancia en terreno (Cob: porcentaje de cobertura; media $\pm \mathrm{EE}$ ) de especies sésiles seleccionadas (ver Métodos); Columnas tres a siete: frecuencia de ocurrencia (\%) por comunidad (nombres abreviados) y a nivel regional; Columnas ocho y nueve: ocurrencia temporal (proporción de estaciones muestreadas; \%) dentro de los períodos de presencia (EN-P) y ausencia (EN-A) del evento El Niño 2004-2005. RS: Río Seco, CC: Caleta Constitución, CA: Caleta Angosta, LA: Lagunillas

Dietary items of Tetrapygus niger in northern Chile, separated by taxonomic group and listed in decreasing order following their regional occurrence frequency. Column two: field abundance (Cob: coverage percentage; mean \pm SE) of selected sessile species (see Methods); Columns three to seven: occurrence frequency (\%) per community (abbreviated names) and at regional level; Columns eight and nine: temporal occurrence (proportion of sampling seasons; \%) within the periods of presence (EN-P) and absence (EN-A) of the 2005-05 El Niño event. RS: Río Seco, CC: Caleta Constitución, CA: Caleta Angosta, LA: Lagunillas.

\begin{tabular}{|c|c|c|c|c|c|c|c|c|}
\hline Ítem dietario & Cob & RS & $\mathrm{CC}$ & $\mathrm{CA}$ & LA & Regional & EN-P & EN-A \\
\hline \multicolumn{9}{|l|}{ Chlorophyta } \\
\hline Ulva rigida $\mathrm{C}$. Agardh & $12,5 \pm 0,7$ & 62,5 & 86,2 & 90,0 & 87,1 & 81,3 & 100 & 100 \\
\hline Enteromorpha sp. & $2,2 \pm 0,5$ & 6,3 & 10,8 & 40,0 & 56,5 & 27,9 & 67 & 58 \\
\hline Pseudoulvella sp. & $10,3 \pm 0,7$ & 17,2 & 10,8 & 31,7 & 24,2 & 20,7 & 17 & 83 \\
\hline Chaetomorpha sp. & $3,8 \pm 1,6$ & 4,7 & 7,7 & 13,3 & 32,3 & 14,3 & 33 & 67 \\
\hline Blidingia sp. & & 0 & 0 & 21,7 & 35,5 & 13,9 & 0 & 42 \\
\hline Codium dimorphum Svedelius & $13,7 \pm 1,6$ & 12,5 & 9,2 & 13,3 & 14,5 & 12,4 & 0 & 33 \\
\hline Cladophora fascicularis (Mertens) Kützing & $5,4 \pm 0,6$ & 0 & 0 & 18,3 & 19,4 & 9,2 & 0 & 17 \\
\hline Rama novazelandiae (J. Agardh) Chapman & & 0 & 7,7 & 8,3 & 14,5 & 7,6 & 0 & 42 \\
\hline Rhizoclonium sp. & $2,9 \pm 1,4$ & 0 & 0 & 10,0 & 14,5 & 6,0 & 8 & 33 \\
\hline Urospora sp. & & 0 & 4,6 & 8,3 & 0 & 3,2 & 8 & 17 \\
\hline Ulothrix sp. & $1,0 \pm 0,0$ & 0 & 4,6 & 0 & 0 & 1,2 & 0 & 8 \\
\hline Chaetomorpha brachygona Harvey & & 0 & 4,6 & 0 & 0 & 1,2 & 0 & 8 \\
\hline Chaetomorpha firma Levring & & 0 & 0 & 1,7 & 0 & 0,4 & 0 & 8 \\
\hline \multicolumn{9}{|l|}{ Phaeophyta } \\
\hline Macrocystis sp. & & 53,1 & 15,4 & 71,7 & 48,4 & 46,6 & 50 & 67 \\
\hline Lessonia nigrescens Bory & & 14,1 & 3,1 & 28,3 & 27,4 & 17,9 & 17 & 33 \\
\hline Halopteris funicularis (Montagne) Sauvegeau & & 0 & 1,5 & 45,0 & 11,3 & 13,9 & 17 & 50 \\
\hline Ectocarpus sp. & $8,3 \pm 3,2$ & 23,4 & 7,7 & 5,0 & 16,1 & 13,1 & 0 & 67 \\
\hline Colpomenia sp. & $5,3 \pm 0,6$ & 23,4 & 6,2 & 13,3 & 8,1 & 12,7 & 33 & 8 \\
\hline Myriogloia sp. & & 0 & 0 & 30,0 & 16,1 & 11,2 & 0 & 33 \\
\hline Glossophora kunthii (C. Agardh) J. Agardh & $4,1 \pm 0,5$ & 6,3 & 12,3 & 6,7 & 0 & 6,4 & 0 & 25 \\
\hline Ectocarpus confervoides (Roth) Le Jolis & & 0 & 0 & 3,3 & 9,7 & 3,2 & 0 & 17 \\
\hline Sphacelaria furcigera Kützing & & 0 & 0 & 10,0 & 1,6 & 2,8 & 0 & 17 \\
\hline Endarachne binghamiae J. Agardh & $4,7 \pm 1,2$ & 0 & 6,2 & 3,3 & 0 & 2,4 & 0 & 17 \\
\hline Ralfsia sp. & $10,0 \pm 1,1$ & 6,3 & 1,5 & 0 & 0 & 2,0 & 0 & 25 \\
\hline Desmarestia sp. & & 0 & 0 & 0 & 3,2 & 0,8 & 8 & 8 \\
\hline Hincksia granulosa (Smith) Silva & & 1,6 & 0 & 0 & 0 & 0,4 & 0 & 8 \\
\hline Petalonia fascia (Müller) Kuntze & $3,5 \pm 1,0$ & 1,6 & 0 & 0 & 0 & 0,4 & 0 & 8 \\
\hline \multicolumn{9}{|l|}{ Rhodophyta } \\
\hline Coralina incrustante & $32,1 \pm 1,4$ & 100,0 & 53,8 & 68,3 & 75,8 & 74,5 & 100 & 92 \\
\hline \multicolumn{9}{|l|}{ Gelidium chilense (Montagne) Santelices et } \\
\hline Montalva & $24,1 \pm 1,4$ & 53,1 & 60,0 & 40,0 & 66,1 & 55,0 & 100 & 100 \\
\hline Hildenbrandia sp. & $9,5 \pm 0,6$ & 28,1 & 38,5 & 46,7 & 43,5 & 39,0 & 67 & 83 \\
\hline Ceramium rubrum (Hudson) C. Agardh & $5,0 \pm 0,9$ & 17,2 & 55,4 & 36,7 & 21,0 & 32,7 & 83 & 58 \\
\hline Polysiphonia sp. & & 17,2 & 23,1 & 35,0 & 37,1 & 27,9 & 83 & 58 \\
\hline \multicolumn{9}{|l|}{ Schottera nicaeensis (Lamouroux ex Duby) } \\
\hline Guiry et Hollenberg & $7,6 \pm 0,9$ & 25,0 & 26,2 & 16,7 & 41,9 & 27,5 & 75 & 83 \\
\hline Hурпеа sp. & $14,1 \pm 1,2$ & 0 & 43,1 & 33,3 & 17,7 & 23,5 & 50 & 58 \\
\hline $\begin{array}{l}\text { Gastroclonium cylindricum Santelices, } \\
\text { Abbott et Ramirez }\end{array}$ & $4,1 \pm 0,9$ & 18,8 & 18,5 & 10,0 & 12,9 & 15,1 & 50 & 58 \\
\hline
\end{tabular}


TABLA 2 (continuación)

\begin{tabular}{|c|c|c|c|c|c|c|c|c|}
\hline Ítem dietario & Cob & RS & $\mathrm{CC}$ & $\mathrm{CA}$ & LA & Regional & EN-P & EN-A \\
\hline Polysiphonia pacifica Hollenberg & $10,5 \pm 1,2$ & 14,1 & 12,3 & 13,3 & 11,3 & 12,7 & 0 & 75 \\
\hline Erytrotrichia $\mathrm{sp}$ & & 1,6 & 4,6 & 21,7 & 22,6 & 12,4 & 17 & 75 \\
\hline Centroceras clavulatum (C. Agardh) Montagne & $16,2 \pm 4,3$ & 7,8 & 24,6 & 3,3 & 8,1 & 11,2 & 17 & 67 \\
\hline Porphyra columbina Montagne & $17,1 \pm 1,3$ & 6,3 & 9,2 & 1,7 & 25,8 & 10,8 & 33 & 42 \\
\hline \multicolumn{9}{|l|}{ Montemaria horridula (Montagne) Joly et } \\
\hline Alveal & $22,0 \pm 3,2$ & 0 & 16,9 & 0 & 24,2 & 10,4 & 33 & 25 \\
\hline Chondrus canaliculatus (C. Agardh) Greville & $5,0 \pm 1,1$ & 12,5 & 7,7 & 8,3 & 4,8 & 8,4 & 8 & 67 \\
\hline Amphiroa dimorpha Dawson & $10,5 \pm 1,3$ & 3,1 & 1,5 & 11,7 & 14,5 & 7,6 & 17 & 50 \\
\hline \multicolumn{9}{|l|}{ Corallina officinalis } \\
\hline (Decaisne) Kützing & $20,1 \pm 3,0$ & 9,4 & 12,3 & 0 & 0 & 5,6 & 25 & 25 \\
\hline Acrochaetium grandis (Levring) Papenfuss & $12,5 \pm 4,7$ & 6,3 & 1,5 & 3,3 & 8,1 & 4,8 & 25 & 25 \\
\hline Plocamium cartilagineum (Linnaeus) Dixon & $3,7 \pm 1,3$ & 0 & 3,1 & 13,3 & 1,6 & 4,4 & 25 & 33 \\
\hline Petroglossum sp. & $12,0 \pm 1,4$ & 0 & 9,2 & 3,3 & 3,2 & 4,0 & 25 & 8 \\
\hline Amphiroa peruana Areschoug & $8,5 \pm 1,4$ & 1,6 & 10,8 & 0 & 0 & 3,2 & 8 & 8 \\
\hline Chondria californica (Collins) Kylin & $6,5 \pm 1,8$ & 0 & 10,8 & 0 & 1,6 & 3,2 & 0 & 25 \\
\hline \multicolumn{9}{|l|}{ Gigartina tuberculosa (Hooker et Harvey) } \\
\hline Grunow & $24,1 \pm 2,3$ & 0 & 12,3 & 0 & 0 & 3,2 & 0 & 8 \\
\hline Gelidium lingulatum Kützing & $7,4 \pm 1,4$ & 0 & 7,7 & 0 & 3,2 & 2,8 & 0 & 17 \\
\hline Polysiphonia mollis Hooker et Harvey & $10,3 \pm 1,8$ & 0 & 7,7 & 0 & 3,2 & 2,8 & 0 & 17 \\
\hline Pterosiphonia pennata (C. Agardh) Sauvageau & $7,6 \pm 2,0$ & 0 & 6,2 & 3,3 & 0 & 2,4 & 8 & 17 \\
\hline Polysiphonia paniculata Montagne & $13,4 \pm 2,3$ & 3,1 & 0 & 5,0 & 0 & 2,0 & 0 & 17 \\
\hline Gelidium pusillum (Stackhouse) Le Jolis & $8,7 \pm 1,3$ & 3,1 & 0 & 3,3 & 0 & 1,6 & 0 & 25 \\
\hline Ceramium pacificum (Collins) Kylin & & 0 & 3,1 & 1,7 & 0 & 1,2 & 0 & 17 \\
\hline Rhodymenia sp. & & 0 & 1,5 & 1,7 & 0 & 0,8 & 0 & 17 \\
\hline Antithamnion densus (Suhr) Howe & & 0 & 1,5 & 0 & 0 & 0,4 & 0 & 8 \\
\hline \multicolumn{9}{|l|}{ Invertebrados } \\
\hline Cirripedia & & 45,3 & 35,4 & 41,7 & 46,8 & 42,2 & 33 & 92 \\
\hline Liopetrolisthes mitra (Dana) & & 26,6 & 23,1 & 21,7 & 33,9 & 26,3 & 67 & 58 \\
\hline Notochthamalus scabrosus (Darwin) & $18,7 \pm 1,3$ & 0 & 10,8 & 23,3 & 32,3 & 16,3 & 8 & 42 \\
\hline Demospongiae & & 10,9 & 9,2 & 10,0 & 1,6 & 8,0 & 8 & 50 \\
\hline Jehlius cirratus (Darwin) & $30,7 \pm 1,6$ & 4,7 & 18,5 & 0 & 8,1 & 8,0 & 17 & 25 \\
\hline Semimytilus algosus (Gould) & $7,7 \pm 2,1$ & 15,6 & 10,8 & 0 & 3,2 & 7,6 & 25 & 17 \\
\hline Spirorbidae & $2,9 \pm 0,7$ & 3,1 & 0 & 5,0 & 12,9 & 5,2 & 0 & 50 \\
\hline Hydrozoa & & 6,3 & 6,2 & 0 & 8,1 & 5,2 & 0 & 25 \\
\hline Sipunculidae & & 18,8 & 0 & 0 & 0 & 4,8 & 0 & 8 \\
\hline Bryozoa & $1,8 \pm 0,4$ & 15,6 & 0 & 0 & 1,6 & 4,4 & 8 & 25 \\
\hline Polychaeta & & 6,3 & 3,1 & 1,7 & 0 & 2,8 & 8 & 25 \\
\hline Balanus flosculus Darwin & $3,8 \pm 0,6$ & 4,7 & 0 & 1,7 & 3,2 & 2,4 & 0 & 25 \\
\hline Phragmatopoma moerchii Kinberg & $4,7 \pm 1,0$ & 0 & 0 & 8,3 & 1,6 & 2,4 & 0 & 17 \\
\hline Perumytilus purpuratus (Lamarck) & $17,0 \pm 2,1$ & 6,3 & 0 & 0 & 1,6 & 2,0 & 8 & 17 \\
\hline Hyale sp. & & 1,6 & 1,5 & 3,3 & 1,6 & 2,0 & 0 & 33 \\
\hline Tegula atra (Lesson) & & 1,6 & 1,5 & 1,7 & 1,6 & 1,6 & 8 & 25 \\
\hline Scurria ceciliana (Orbigny) & & 4,7 & 0 & 0 & 0 & 1,2 & 17 & 8 \\
\hline Isopoda & & 3,1 & 0 & 0 & 1,6 & 1,2 & 0 & 17 \\
\hline Littorina araucana Orbigny & & 3,1 & 0 & 0 & 0 & 0,8 & 0 & 8 \\
\hline Radiolaria & & 0 & 0 & 0 & 3,2 & 0,8 & 0 & 8 \\
\hline Crassilabrum crassilabrum Sowerby & & 1,6 & 0 & 0 & 0 & 0,4 & 0 & 8 \\
\hline Echinolittorina peruviana (Lamarck) & & 1,6 & 0 & 0 & 0 & 0,4 & 8 & 0 \\
\hline Prisogaster niger (Wood) & & 0 & 1,5 & 0 & 0 & 0,4 & 0 & 8 \\
\hline Chironomidae & & 0 & 0 & 0 & 1,6 & 0,4 & 8 & 0 \\
\hline Foraminifera & & 1,6 & 0 & 0 & 0 & 0,4 & 0 & 8 \\
\hline Nudibranchia & & 0 & 0 & 0 & 1,6 & 0,4 & 8 & 0 \\
\hline Scurria sp. & & 0 & 0 & 1,7 & 0 & 0,4 & 0 & 8 \\
\hline Copepoda & & 0 & 0 & 0 & 1,6 & 0,4 & 0 & 8 \\
\hline Nematoda & & 0 & 1,5 & 0 & 0 & 0,4 & 0 & 8 \\
\hline Diloma nigerrima (Gmelin) & & 0 & 0 & 0 & 1,6 & 0,4 & 0 & 8 \\
\hline Ostracoda & & 0 & 0 & 0 & 1,6 & 0,4 & 0 & 8 \\
\hline Acanthocyclus gayi (M. Edwards et Lucas) & & 0 & 1,5 & 0 & 0 & 0,4 & 0 & 8 \\
\hline
\end{tabular}


el análisis de estos últimos valores no reveló diferencias significativas entre las comunidades $\left(\mathrm{F}_{3,20}=0,65 ; \mathrm{P}=0,595\right)$, aunque estos datos incluyen la variación estacional y no se descarta un fenómeno subyacente asociado a la latitud.

Al analizar la variación espacio-temporal del consumo a nivel individual (Fig. 2), la riqueza dietaria por comunidad y estación fluctuó entre aproximadamente cuatro y 15 ítemes por individuo en promedio, con una tendencia hacia valores en general más bajos en Río Seco y más altos en Lagunillas. Hubo diferencias estadísticas entre comunidades $\left(\mathrm{F}_{3,15}=5,11 ; \mathrm{P}=\right.$ $0,0124)$ y entre estaciones $\left(\mathrm{F}_{5,227}=70,14 ; \mathrm{P}<\right.$ $0,00001)$, pero también una interacción significativa entre ambos factores $\left(\mathrm{F}_{15,227}=\right.$ $7,17 ; \mathrm{P}<0,00001)$. Sin embargo la interacción no revela una covariación muy coherente entre los factores, y aparentemente se debería a los cambios relativos de los valores de riqueza entre las comunidades a través del tiempo. La Fig. 2 muestra que el patrón más consistente entre comunidades fue el cambio temporal desde niveles de riqueza dietaria comparativamente menores y similares entre invierno 2004 y verano 2005, hacia niveles crecientes a partir de otoño 2005 en Caleta Constitución, Caleta Angosta y Lagunillas, y desde invierno 2005 en Río Seco. La comparación a posteriori indica por una parte la ausencia de diferencias estadísticas entre comunidades dentro de cada período (prueba de Tukey, $\mathrm{P}>0,05$ en todos los casos), y por otra parte un aumento significativo en cada comunidad en las estaciones en que se produjo respectivamente el cambio de tendencia (prueba de Tukey, $\mathrm{P}<0,05$ en todos los casos). En este contexto es pertinente notar la extensión temporal del evento El Niño ocurrido entre agosto 2004 y marzo 2005 .

Al respecto, la riqueza dietaria por estación mostró una asociación negativa y significativa con la TSM (Fig. 3; $\mathrm{r}_{\mathrm{s}}=-0,56 ; \mathrm{n}=24 ; \mathrm{P}=$ $0,005)$, pero no se correlacionó con la biomasa $\left(\mathrm{r}_{\mathrm{s}}=-0,31 ; \mathrm{n}=24 ; \mathrm{P}=0,140\right)$ o la longitud $\left(\mathrm{r}_{\mathrm{s}}\right.$ $=-0,29 ; \mathrm{n}=24 ; \mathrm{P}=0,165)$ de los individuos. Sin embargo, al desglosar componentes de la dieta se detectó una correlación negativa y significativa del número de ítemes animales con la biomasa $\left(\mathrm{r}_{\mathrm{s}}=-0,52 ; \mathrm{n}=24 ; \mathrm{P}=0,0009\right)$ y la longitud $\left(\mathrm{r}_{\mathrm{s}}=-0,55 ; \mathrm{n}=24 ; \mathrm{P}=0,010\right) \mathrm{de}$ T. niger.

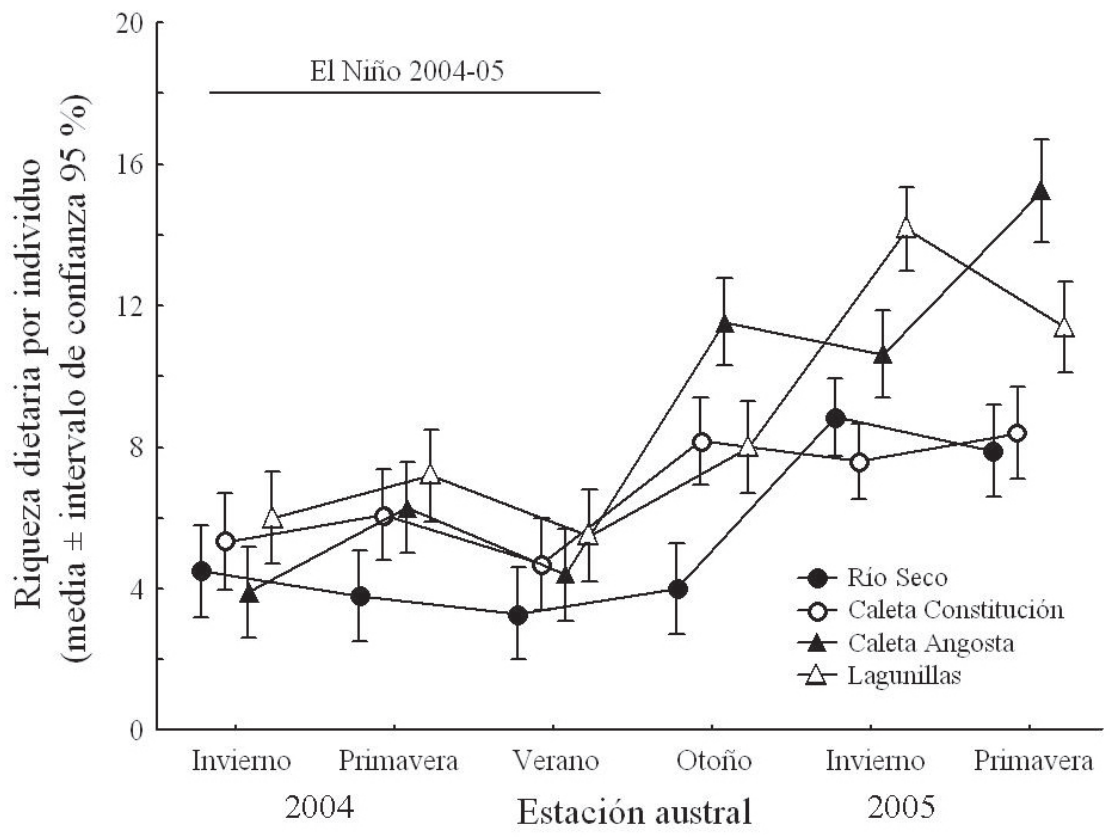

Fig. 2: Variación estacional de la riqueza dietaria por individuo de Tetrapygus niger en las comunidades de estudio. La línea horizontal indica la extensión temporal del evento El Niño 2004-2005. Valores: media \pm intervalo de confianza del $95 \%$.

Seasonal variation of diet richness per individual of Tetrapygus niger in the study communities. The horizontal line marks the temporal extent of the 2005-2005 El Niño event. Values: mean $\pm 95 \%$ confidence interval. 
En relación a la oferta ambiental y el consumo de recursos a nivel regional, la relación entre cobertura promedio en terreno y frecuencia de ocurrencia en la dieta evaluada para 47 especies sésiles no fue significativa $\left(r_{s}=0,257 ; P=0,081\right)$. Los valores mostraron una alta dispersión, pero notamos que el signo positivo de $r_{s}$ y su probabilidad relativamente baja fueron al parecer forzados por solo tres puntos extremos ("outliers"), con valores altos y muy distanciados de las demás especies. Estos ítemes correspondieron a Gelidium chilense, coralinas incrustantes y Ulva rigida, donde esta última mostró una frecuencia dietaria comparativamente mayor que su cobertura, mientras los dos primeros mostraron valores similarmente altos (Tabla 2). Al analizar separadamente los ítemes dietarios más importantes, en base a su frecuencia dietaria y cobertura en el ambiente evaluadas en cada estación de muestreo, Enteromorpha sp., Gelidium chilense, coralinas incrustantes y cirripedios no mostraron asociaciones significativas $\left(-0,33<\mathrm{r}_{\mathrm{s}}<0,27 ; 0,106<\mathrm{P}<\right.$ $0,530 ; \mathrm{n}=24)$. La única asociación importante fue para Ulva rigida $\left(\mathrm{r}_{\mathrm{s}}=0,404 ; \mathrm{P}=0,050 ; \mathrm{n}=\right.$ $24)$, que fue el recurso algal más frecuente en la dieta superando incluso a las algas Laminariales (Tabla 2).

\section{Composición taxonómica de la dieta}

La composición taxonómica de la dieta de $T$. niger mostró variaciones en espacio y tiempo (nMDS; estrés $=0,2)$, con diferencias significativas entre comunidades (ANOSIM; R global $=0,19 ; \mathrm{P}=0,007) \mathrm{y}$ estaciones $(\mathrm{R}$ global $=0,63 ; \mathrm{P}=0,001)$. El principal contraste fue generado por la comunidad de Río Seco la cual reveló diferencias con todas las demás (pruebas pareadas ANOSIM; 0,31 $<\mathrm{R}<0,44 ; 0,003<\mathrm{P}$ $<0,025)$. Estacionalmente, la mayoría de las comparaciones pareadas revelaron diferencias significativas $(\mathrm{R}>0,46$ y $\mathrm{P}=0,029)$ exceptuando las combinaciones otoño-invierno $2005(\mathrm{R}=0,28 ; \mathrm{P}=0,086)$ e inviernoprimavera $2005(\mathrm{R}=0,14 ; \mathrm{P}=0,20)$.

Lo anterior sugirió que la mayor variación dietaria fue coincidente con el período del evento El Niño 2004-2005, lo cual fue reforzado por las diferencias significativas $(\mathrm{R}$ global $=$ $0,53 ; \mathrm{P}=0,001)$ detectadas en presencia $\mathrm{y}$ ausencia de El Niño (Fig. 4). Al revisar cualitativamente estos cambios se encontró que

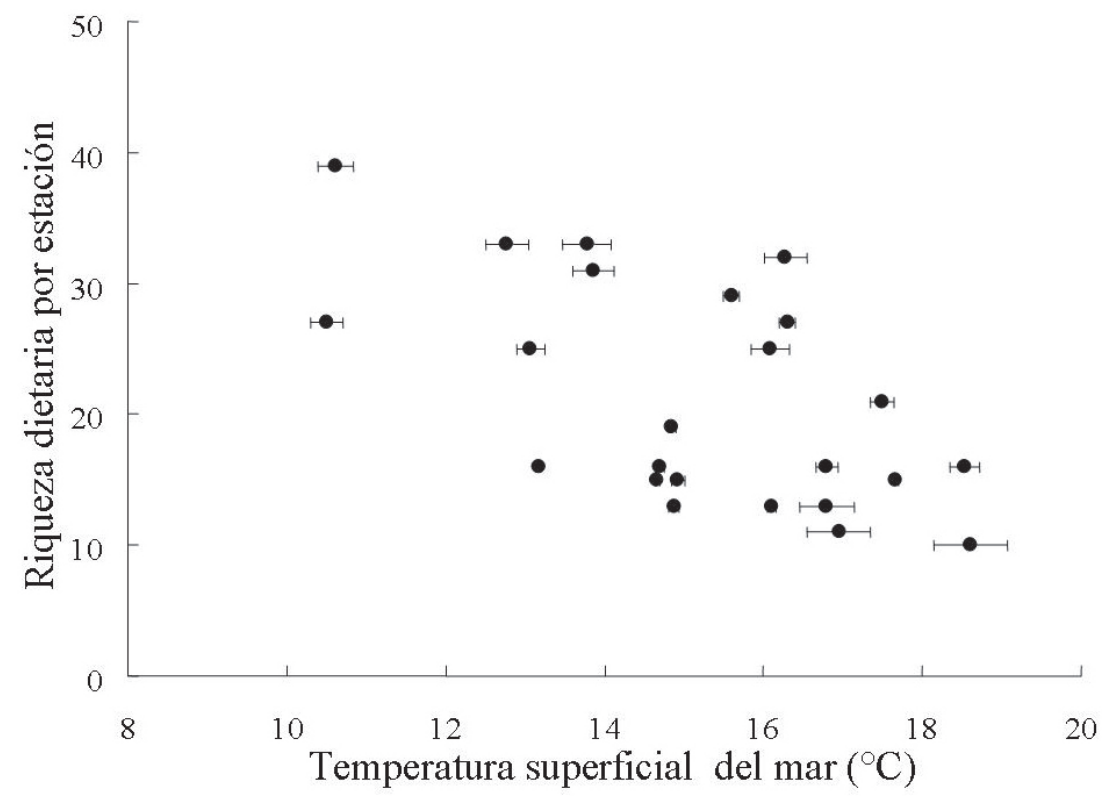

Fig. 3: Relación entre la riqueza dietaria de Tetrapygus niger por estación (comunidades combinadas) con la temperatura superficial del mar (media \pm EE) medida in situ.

Relationship between dietary richness of Tetrapygus niger per season (combined communities) and sea surface temperature $($ mean $\pm \mathrm{SE})$ measured in situ. 
los ítemes consumidos por T. niger durante $\mathrm{El}$ Niño fueron esencialmente los mismos que en ausencia de El Niño, y la diferencia se debió a un aumento significativo del número de ítemes dietarios desde 45 a 86 respectivamente, el cual se reflejó en todos los grupos taxonómicos mayores: de 20 a 30 ítemes de Rodhophyta, seis a 13 de Chlorophyta, cinco a 14 de Phaeophyta, y 14 a 29 de invertebrados. El único cambio real en composición dietaria fue el consumo de tres ítemes animales (nudibranquios, insectos quironómidos y el gastrópodo pulmonado Echinolittorina peruviana) registrados solo durante el período El Niño. Las variaciones anteriores están registradas en la Tabla 2, que muestra la proporción de estaciones en que cada ítem estuvo presente en la dieta durante los períodos de presencia y ausencia del evento El Niño 2004-2005.

\section{DISCUSIÓN}

\section{El rol de Tetrapygus niger como consumidor}

En el norte de Chile, el erizo negro T. niger muestra un comportamiento trófico relativamente homogéneo a nivel regional, aparentemente con baja dependencia de la oferta ambiental, y baja influencia de factores locales. Además, T. niger es sin duda un consumidor principalmente herbívoro pero también un generalista polífago, como demuestran sus 89 ítemes dietarios, y por tanto un omnívoro de acuerdo a una definición trófica de uso frecuente (consumir presas en más de un nivel trófico, Pimm \& Lawton 1978). En este sentido, es sugerente que $36 \%$ de los ítemes dietarios fueran invertebrados, y que los individuos de menor tamaño los consuman en mayor proporción, independientemente de las variaciones en el tamaño medio de $T$. niger entre comunidades. Como se indicó anteriormente, la omnivoría en erizos no es rara, y se sabe que algunas especies aumentan significativamente su producción gonádica ingiriendo ítemes animales (e.g., Knip \& Scheibling 2007). Por ello es necesario evaluar experimentalmente la opción de que $T$. niger digiera y asimile los ítemes animales que ingiere, y la posible relación nutricional de este consumo con la reproducción y variaciones ontogenéticas en la dieta.

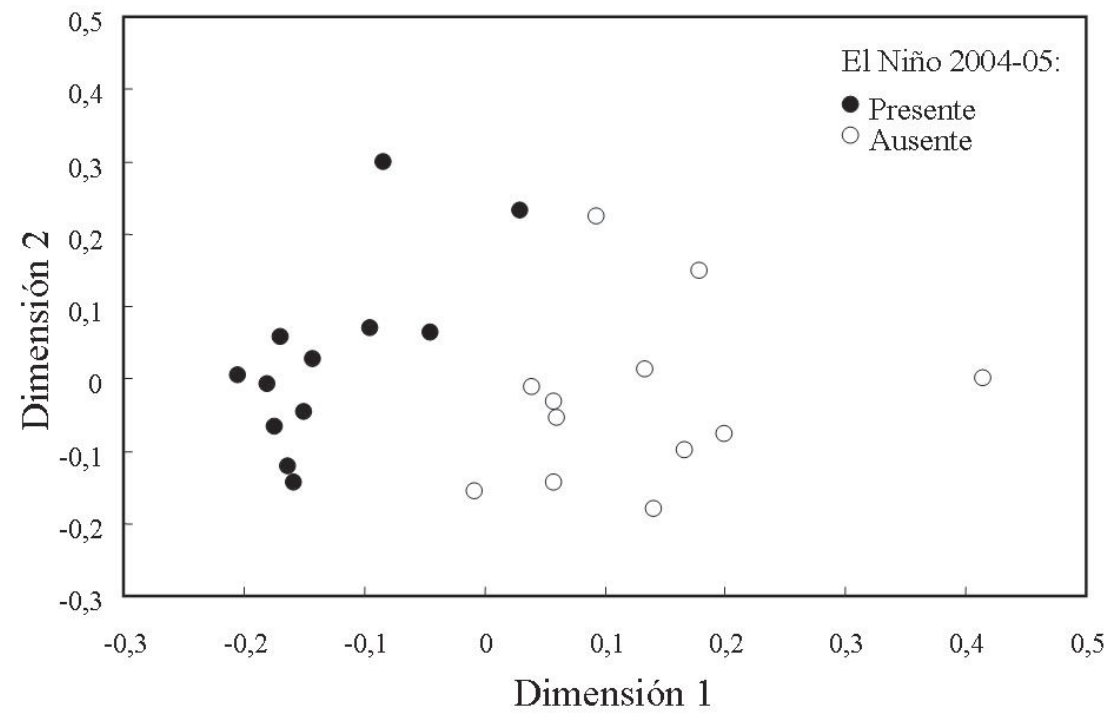

Fig. 4: Ordenación (nMDS) de la composición taxonómica de la dieta de Tetrapygus niger registrada en 24 combinaciones muestrales de comunidad x estación, clasificadas de acuerdo a la presencia (negro) o ausencia (blanco) del evento El Niño 2004-2005.

Ordination (nMDS) of the taxonomic composition of the diet of Tetrapygus niger recorded in 24 sampling combinations of community $\mathrm{x}$ season, classified according with the presence (filled dots) or absence (open dots) of the 2004-2005 El Niño event. 
Por otra parte, T. niger también ingiere a otros herbívoros con los que a su vez puede compartir recursos algales, lo cual fuerza dos consideraciones adicionales sobre su rol trófico en el ecosistema litoral. Primero, esto posiciona a $T$. niger en conceptos de omnivoría más precisos, como estar ligado a una especie basal a más de un link de distancia en una cadena trófica (Yodzis 1984), o consumir especies basales e intermedias (Arim \& Marquet 2004). Segundo, T. niger presenta una superposición dietaria importante con los herbívoros ingeridos, convirtiéndose en un potencial depredador intragremio además de omnívoro (Camus et al. 2008). Lo anterior permite hipotetizar que $T$. niger podría contribuir de modo importante a la conectividad y complejidad de la trama trófica participando en loops omnívoros (e.g., Polis et al. 1989), generando efectos indirectos, y agregando links débiles que pueden ser relevantes para la estabilidad de la trama (e.g., Neutel et al. 2002). De este modo, T. niger podría ser más que un simple herbívoro, y aun cuando las interacciones con sus recursos implicaran bajos efectos per cápita, es pertinente notar que varios de los herbívoros intermareales más comunes desarrollan interacciones similares (Camus et al. 2008), lo cual sugiere que nuestra visión corriente de estos herbívoros debiera cuando menos expandirse.

Si bien $T$. niger efectivamente ingiere invertebrados y otros herbívoros, notamos sin embargo que esto no implica que persigan, ataquen o capturen activamente estas presas como hacen otros predadores carnívoros, y de hecho parte de este consumo podría ser solo incidental, particularmente el de aquellos ítemes misceláneos ingeridos en baja frecuencia. Por otra parte, nuestros datos no permiten demostrar que los ítemes animales consumidos representen una fuente nutricional para $T$. niger, lo cual debiera evaluarse en forma directa (e.g., mediante isótopos estables u otros marcadores, usados para evaluar posición trófica y omnivoría en consumidores acuáticos, Lancaster et al. 2005, Latyshev et al. 2005). No obstante, la eurifagia de tipo omnívora y la depredación intragremio aparente observadas en $T$. niger también son comunes en otros herbívoros intermareales importantes como Loxechinus albus y las especies de chitones y fisurélidos de mayor tamaño (Camus et al. 2008). De hecho análisis preliminares en Chiton granosus Frembly, Fissurella picta (Gmelin) y Fissurella limbata Sowerby (Cid 20071․, Cisterna 2007²), indican la existencia de actividad proteolítica y asimilación efectiva de alimento animal por estas especies. Si bien se espera confirmar estos resultados con ensayos más completos, y extendiéndolos a otos herbívoros como $T$. niger, no es improbable que la omnivoría sea un fenómeno real y común en los consumidores intermareales.

\section{Variación dietaria y factores ambientales}

La disminución en amplitud del nicho dietario de $T$. niger hacia menores latitudes fue un resultado sugerente, ya que parece responder a la predicción clásica de MacArthur (1972) basada en la disminución en estacionalidad, un supuesto aumento en estabilidad hacia los trópicos, y la consecuente especialización de los consumidores. Estos fundamentos han sido cuestionados y replanteados, pero la predicción aún es válida en base a la simetría y anidamiento de las interacciones interespecíficas, donde una condición necesaria es la existencia de un gradiente en riqueza de especies (Vásquez \& Stevens 2004). Si bien tal gradiente existe, es inverso al requerido por la hipótesis ya que la riqueza de herbívoros intermareales disminuye desde alrededor de los $30^{\circ} \mathrm{S}$ hacia el norte (Rivadeneira et al. 2002). Por tanto, la tendencia latitudinal en el nicho de T. niger puede ser solo un artefacto, pero también un fenómeno real no asociado a la especialización trófica y que requiere mayor estudio. En forma independiente de esta tendencia, nuestros resultados muestran que la amplitud de nicho experimenta una variación temporal importante, y probablemente relacionada a las determinantes físicas de gran

1 CID Y (2007) Evaluación de la asimilación de alimento animal en Chiton granosus (Frembly, 1827) y su estrategia trófica como potencial omnívoro. Seminario de investigación, Facultad de Ciencias, Universidad Católica de la Santísima Concepción, Concepción, Chile. 13 pp.

2 CISTERNA L (2007) Potencial de omnivoría en dos especies del género Fissurella: evaluación experimental de la capacidad de digestibilidad de alimento animal. Seminario de investigación, Facultad de Ciencias, Universidad Católica de la Santísima Concepción, Concepción, Chile. 25 pp. 
escala sobre la variabilidad ambiental en el norte de Chile (Thiel et al. 2007).

Al comparar los registros temporales de la dieta en las comunidades de estudio, nuestros resultados mostraron que en las fechas posteriores al evento El Niño 2004-2005 el número de ítemes consumidos por $T$. niger de cada grupo taxonómico mayor incrementó entre 150 y $280 \%$, causando un aumento global de $191 \%$ en el espectro dietario completo. No obstante, este estudio abarcó un solo evento El Niño, y existe una alta variación entre eventos en su intensidad, duración, características físicas y efectos biológicos (Kerr 1999, Larkin \& Harrison 2002, Thiel et al. 2007, Camus 2008), y es factible que los patrones observados se deban en menor o mayor grado a otro tipo de factores (Camus 2008). Ya que la información anterior no constituye prueba y solo muestra un efecto putativo de El Niño, planteamos como hipótesis que El Niño limita o al menos reduce la disponibilidad de recursos para consumidores como $T$. niger, generando una reducción significativa de su espectro dietario. Esta hipótesis es respaldada por otros aspectos como la relación negativa entre la riqueza dietaria de T. niger y la TSM detectada en este estudio, o reducciones similares en riqueza dietaria observadas en otros herbívoros asociadas al mismo evento El Niño (Navarrete et al. $2007^{3}$, Camus 2008). Por otra parte, $T$. niger también incorporó más presas animales durante El Niño, lo que podría vincularse a la falta de otros recursos, pero un análisis detallado del consumo de invertebrados por las especies herbívoras intermareales de la zona norte (Camus et al. 2008) no indica relaciones claras entre este fenómeno y una eventual limitación de recursos. En el mismo sentido, la variación dietaria de $T$. niger no mostró asociación evidente con la surgencia costera, y por ejemplo en el período posterior a El Niño la riqueza dietaria en Río Seco y Caleta Angosta (sin surgencia) fue mayor o al menos tan alta como en Caleta Constitución y Lagunillas (con surgencia). Si bien estudios en Chile central

\footnotetext{
3 NAVARRETE A, F Opazo \& PA Camus (2007) Variación espacio-temporal en patrones tróficos de herbívoros intermareales del norte de Chile. III Reunión Binacional de Ecología, Sociedad de Ecología de Chile y Asociación Argentina de Ecología, La Serena, Chile. Resumen 324.
}

muestran que la variación en intensidad de surgencia afecta diferencialmente la abundancia de distintos tipos de algas (Nielsen \& Navarrete 2004), nuestros resultados, al menos a nivel de la dieta de herbívoros, sugieren que en el norte de Chile los efectos El Niño podrían neutralizar o suprimir el efecto de la surgencia, y/o que la surgencia no sería una determinante central al explicar las variaciones dietarias. Los patrones contrastantes en la Fig. 2 entre presencia y ausencia de El Niño sugieren que ambas explicaciones son factibles, y reforzadas por los resultados asociados a la Fig. 4.

Por otra parte, nuestros datos también indican que no hay una relación clara entre el consumo y la oferta ambiental de recursos, sugiriendo que si bien $T$. niger es claramente un generalista trófico, no necesariamente actúa en forma oportunista. En este contexto destaca el caso de Ulva rigida, una especie común en la zona norte pero no la más abundante (véase Tabla 2), pero que en todas las comunidades fue consumida con más alta frecuencia que cualquier otro ítem algal o animal. De hecho su mayor frecuencia de consumo se registró en Caleta Angosta, un sitio con escaso aporte de surgencia, aunque se ha mostrado que la producción de biomasa por unidad de cobertura de Ulva es mayor en lugares del norte de Chile con surgencia intensa ( Constitución, Camus \& Andrade 1999). Sin embargo, es interesante notar que en Chile central la tasa de crecimiento de Gelidium chilense (importante en la dieta de T. niger en el norte) sea mayor en sitios con surgencia intensa (Wieters 2005), y en Chile norte su consumo fuera coincidentemente más frecuente en los sitios comparables (Lagunillas y Caleta Constitución). Lo anterior sugiere que T. niger podría aprovechar de modo oportunista el efecto positivo de la surgencia sobre recursos como G. chilense, cuya abundancia puede ser poco afectada por eventos El Niño, mientras a la vez podría preferir otros como $U$. rigida independientemente de cómo sean afectados por la variación física ambiental.

\section{AGRADECIMIENTOS}

Este trabajo fue financiado por el proyecto FONDECYT 1040425. Agradecemos la asistencia en laboratorio y terreno por Daniela 
López, Karen Daroch y Christian Ibáñez, así como los útiles comentarios de dos revisores anónimos.

\section{LITERATURA CITADA}

ARANA P (2005) Demografía y pesquería del erizo Loxechinus albus (Echinodermata: Echinidae) en la región sur-austral de Chile. Revista de Biología Tropical 53: 367-382.

ARIM M \& PA MARQUET (2004) Intraguild predation: a widespread interaction related to species biology. Ecology Letters 7: 557-564.

BRISCOE CS \& KP SEBENS (1988) Omnivory in Strongylocentrotus droebachiensis (Müller) (Echinodermata: Echinoidea): predation on subtidal mussels. Journal of Experimental Marine Biology and Ecology 115: 1-24.

BUSTAMANTE RH, GM BRANCH, S EEKHOUT, B ROBERTSON, P ZOUTENDYK, M SCHLEYER, A DYE, N HANEKOM, D KEATS, M JURD \& C McQUAID (1995) Gradients of intertidal primary productivity around the coast of South Africa and their relationships with consumer biomass. Oecologia 102: 189-201.

CAMUS PA (en prensa) Understanding biological impacts of ENSO on the eastern Pacific: An evolving scenario. International Journal of Environment and Health.

CAMUS PA \& YN ANDRADE (1999) Diversidad de comunidades intermareales rocosas del norte de Chile: el supuesto del enriquecimiento por efecto de la surgencia costera. Revista Chilena de Historia Natural 72: 389-410.

CAMUS PA, K DAROCH \& LF OPAZO (en prensa) Potential for omnivory and apparent intraguild predation in rocky intertidal herbivore assemblages from northern Chile. Marine Ecology Progress Series.

CASTILLA JC \& C MORENO (1982) Sea urchin and Macrocystis pyrifera. Experimental test of their ecological relations in southern Chile. En: Lawrence JM (ed) International ehinoderms conference, Tampa Bay: 257-263. A.A. Balkema, Rotterdam, The Netherlands.

CASTILLA JC \& M FERNÁNDEZ (1998) Small-scale benthic fisheries in Chile: on co-management and sustainable use of benthic invertebrates. Ecological Applications 8 (Supplement): 124-132.

CONTRERAS S \& JC CASTILLA (1987) Feeding behaviour and morphological adaptations in two sympatric sea urchin species in central Chile. Marine Ecology Progress Series 38: 217-224.

CPC (2007) Cold \& warm episodes by season. Climate Prediction Center, NOAA, USA, w w. c p c.nce p.noa a.gov/product s/ analysis_monitoring/ensostuff/ensoyears.shtml

FARIÑA JM, AT PALMA \& FP OJEDA (en prensa) A food web perspective of the subtidal communities off the temperate Chilean coast. En: McClanahan TR \& GM Branch (eds) Food webs and trophic dynamics of marine benthic ecosystems. Oxford University Press, United Kingdom.

HAROLD C \& JS PEARSE (1987) The ecological role of echinoderms in kelps forest. En: Jangoux M \& JM Lawrence (eds) Echinoderm studies: 2137-233. A.A. Balkema, Rotterdam, The Netherlands.
HAWKINS SJ \& RG HARTNOLL (1983) Grazing of intertidal algae by marine invertebrates. Oceanography and Marine Biology: an Annual Review 21: 195-282.

HUGHES AD, AI CATARINO, MS KELLY, DKA BARNES \& KD BLACK (2005) Gonad fatty acids and trophic interactions of the echinoid Psammechinus miliaris. Marine Ecology Progress Series 305: 101-111.

KERR RA (1999) Does a globe-girdling disturbance jigger El Niño? Science 285: 322-323

KNIP DM \& RE SCHEIBLING (2007) Invertebrate fauna associated with kelp enhance reproductive output of green sea urchin Strongylocentrotus droebachiensis. Journal of Experimental Marine Biology and Ecology 351: 150-159.

LANCASTER J, DC BRADLEY, A HOGAN \& S WALDRON (2005) Intraguild omnivory in predatory stream systems. Journal of Animal Ecology 74: 619-629.

LARKIN NK \& DE HARRISON (2002) ENSO warm (El Niño) and cold (La Niña) event life cycles: ocean surface anomaly patterns, their symmetries, asymmetries, and implications. Journal of Climate 15: 1118-1140.

LATYSHEV NA, AS KHARDIN, SP KASYANOV \& MB IVANOVA (2004) A study in the feeding ecology of chitons using analysis of gut contents and fatty acid markers. Journal of Molluscan Studies 70: 225230 .

LAWRENCE JM (1975) On the relationships between marine plants and sea urchins. Oceanography and Marine Biology: an Annual Review 13: 213-286.

LAWRENCE JM \& P SAMMARCO (1982) Effects of feeding on the environment: Echinoidea. En: Jangoux M \& JM Lawrence (eds) Echinoderm nutrition: 499-519. A.A. Balkema, Rotterdam, The Netherlands

LEVINS R (1968) Evolution in changing environments: some theoretical explorations. Princeton University Press, Princeton, New Jersey, USA. 120 pp.

MacARTHUR RH (1972) Geographical ecology: patterns in the distribution of species. Princeton University Press, Princeton, New Jersey, USA. 288 pp.

NEUTEL A-M, JAP HEESTERBEEK \& PC DE RUITER (2002) Stability in real food webs: weak links in long loops. Science 296: 1120-1123.

NIELSEN KJ \& SA NAVARRETE (2004) Mesoscale regulation comes from the bottom-up: intertidal interactions between consumers and upwelling. Ecology Letters 7: 31-41.

PIMM SL \& JH LAWTON (1978) On feeding on more than one trophic level. Nature 275: 542-544.

POLIS GA, CA MYERS \& RD HOLT (1989) The ecology and evolution of intraguild predation: potential competitors that eat each other. Annual Review of Ecology and Systematics 20: 297-330.

RIVADENEIRA MM, M FERNÁNDEZ \& SA NAVARRETE (2002) Latitudinal trends of species diversity in rocky intertidal herbivore assemblages: spatial scale and the relationship between local and regional species richness. Marine Ecology Progress Series 235: 123-131.

RODRÍGUEZ SR (2003) Consumption of drift kelp by intertidal populations of the sea urchin Tetrapygus niger on the central Chilean coast: possible consequences at different ecological levels. Marine Ecology Progress Series 251: 141-151.

SANTELICES B \& FP OJEDA (1984) Recruitment, growth and survival of Lessonia nigrescens 
(Phaeophyta) at various tidal levels in exposed habitats of central Chile. Marine Ecology Progress Series 19: 73-82.

SIEGEL S \& J CASTELLAN (1988) Nonparametric statistics for the behavioral sciences. Second edition. McGraw-Hill, New York, New York, USA. $339 \mathrm{pp}$.

STOTZ W, SA GONZÁLEZ \& C LÓPEZ (1992) Repoblación experimental del erizo rojo Loxechinus albus Molina en la costa expuesta del centro-norte de Chile: efectos del erizo negro Tetrapygus niger Molina sobre el establecimiento y crecimiento de los juveniles. Investigaciones Pesqueras (Chile) 37: 107-117.

THIEL M, E MACAYA, E ACUÑA, W ARNTZ, H BASTÍAS, K BROKORDT, PA CAMUS, JC CASTILLA, LR CASTRO, M CORTÉS, CP DUMONT, R ESCRIBANO, M FERNÁNDEZ, DA LANCELLOTTI, JA GAJARDO, CF GAYMER, I GÓMEZ, AE GONZÁLEZ, HE GONZÁLEZ, PA HAYE, JE ILLANES, JL IRIARTE, G LUNAJORQUERA, C LUXORO, PH MANRÍQUEZ, V MARÍN, P MUÑOZ, SA NAVARRETE, E PÉREZ, E POULIN, J SELLANES, A SEPÚLVEDA, W STOTZ, F TALA, A THOMAS, CA VARGAS, JA VÁSQUEZ \& A VEGA (2007) The Humboldt Current System of northern and central Chile: oceanographic processes, ecological interactions and socioeconomic feedback. Oceanography and Marine Biology: an Annual Review 45: 195-345.

VÁSQUEZ DP \& ED STEVENS (2004) The latitudinal gradient in niche breadth: Concepts and evidence. American Naturalist 164: 1-19.

VÁSQUEZ JA (2000) Ecology of Loxechinus albus. En: Lawrence $J$ (ed) Biology and ecology of edible sea urchins: 161-175. Elsevier B.V., Amsterdam, The Netherlands.

VÁSQUEZ JA \& A BUSCHMANN (1997) Herbivory-kelp interactions in subtidal chilean communities: a review. Revista Chilena de Historia Natural 70: 4152.

VÁSQUEZ JA, JMA VEGA \& AH BUSCHMANN (2006) Long term variability in the structure of kelp communities in northern Chile and the 1997-98 ENSO. Journal of Applied Phycology 18: 505-519.

VEGA JMA, JA VÁSQUEZ \& AH BUSCHMANN (2005) Biología poblacional de huirales submareales de Macrocystis integrifolia y Lessonia trabeculata (Laminariales, Phaeophyceae) en un ecosistema de surgencia del norte de Chile: variabilidad interanual y El Niño 1997-1998. Revista Chilena de Historia Natural 78: 33-50.

WIETERS EA (2005) Upwelling control of positive interactions over mesoscales: a new link between bottom-up and top-down processes on rocky shores. Marine Ecology Progress Series 301: 43-54.

WINEMILLER KO, ER PIANKA, LJ VITT \& A JOERN (2001) Food web laws or niche theory? Six independent empirical tests. American Naturalist 158: 193-199.

YODZIS P (1984) How rare is omnivory? Ecology 65: 321-323. 
